\title{
Tables of Reductions of Symmetrized Inner Products ("Inner Plethysms") of Ordinary Irreducible Representations of Symmetric Groups
}

\author{
By N. Esper
}

\begin{abstract}
Decompositions of symmetrized inner products $[\alpha] \square[\beta]$ of ordinary irreducible representations $[\alpha]$ of symmetric groups $S_{n}$ and $[\beta]$ of $S_{m}$ were evaluated on a CDC 6400. Tables were obtained for $2 \leqslant n \leqslant 10$ and $2 \leqslant m \leqslant 5$ as well as for $m=6$ and $2 \leqslant n \leqslant 7$.
\end{abstract}

In [3] R. C. King published tables of reductions of symmetrized inner products $[\alpha] \bullet[\beta]$ which he calls inner plethysms, of ordinary irreducible representations $[\alpha]$ of $S_{n}$ and $[\beta]$ of $S_{m}$, where $n=4$ and $m \leqslant 5, n=5$ and $m \leqslant 4, n=6$ and $m \leqslant 3$.*

He obtained the decomposition by restricting certain representations $\{\beta\}$ of the general linear group $G L_{n}$ to symmetric subgroups.

Such decompositions can be obtained directly by evaluating the character of $[\alpha] \otimes[\beta]$ which is

$$
\chi^{[\alpha] \varpi[\beta]}(g)=\frac{1}{\left|S_{m}\right|} \sum_{\pi \in S_{m}} \zeta^{[\beta]}(\pi) \prod_{k=1}^{n} \zeta^{[\alpha]}\left(g^{k}\right)^{a_{k}(\pi)},
$$

where $g \in S_{n}$ and $a_{k}(\pi)$ denotes the number of cyclic factors of length $k$ in $\pi \in S_{m}$, $1 \leqslant k \leqslant m$.

For this formula see [1], [2], and [4, p. 74]. The evaluation was carried out with the aid of a computer (CDC 6400 RWTH Aachen) by using the program described in $[1]$, in double-precision arithmetic. The characters of the products $[\alpha] \square[\beta]$ were then decomposed into their irreducible constituents via orthogonality relations by using the character table of $S_{n}$.

Tables were thus obtained of the reductions of the symmetrized inner products of the ordinary irreducible representations of the symmetric groups $S_{2}$ up to $S_{10}$ with the ordinary irreducible representations of $S_{2}$ up to $S_{5}$ and of the characters of $S_{2}$ up to $S_{7}$ with those of $S_{6}$. These tables appear on the microfiche card in this issue.

Acknowledgment. The author wishes to thank Professor A. Kerber for his help and advice.

Received October 18, 1974. $20 \mathrm{C} 04$.

AMS (MOS) subject classifications (1970). Primary 20C30, 20C35, 81 A78; Secondary 05A15,

*Dr. King wants me to point out that in the tables of [3] two printing errors occurred in the decomposition of $[3,1] \cup\left[2^{2}\right]$ and $\left[3,1^{2}\right] \boxminus\left[2,1^{2}\right]$. The correct values can be obtained from the microfiche. 
Lehrstuhl D für Mathematik

RWTH Aachen

Aachen, Federal Republic of Germany

1. N. ESPER, Ein interaktives Programmsystem zur Erzeugung der rationalisierten Charakterentafel einer endlichen Gruppe, Staatsexamensarbeit, Aachen, 1974. (To appear.)

2. A. KERBER, "Symmetrization of representations," Proc. Second Internat. Colloq. Group Theoretical Methods in Physics, Nijmegen, June 1973.

3. R. C. KING, "Branching rules for $\operatorname{GL}(N) \supset \Sigma_{m}$ and the evaluation of inner plethysms," J. Mathematical Phys., v. 15, 1974, pp. 258-267. MR 48 \#10331.

4. G. Ja. LJUBARSKII, Group Theory and Its Applications to Physics, GITTL, Moscow, 1957; English transl., Pergamon Press, New York, 1960. MR 21 \#5441; 22 \#7709. 